\title{
Fibroblast activation protein- $\alpha$ promotes the growth and migration of lung cancer cells via the PI3K and sonic hedgehog pathways
}

\author{
JUN JIA ${ }^{1}$, TRACEY A. MARTIN ${ }^{2}$, LIN YE $^{2}$, LIN MENG $^{3}$, NAN XIA $^{4}$, WEN G. JIANG ${ }^{2}$ and XIAODONG ZHANG ${ }^{1}$ \\ ${ }^{1}$ VIP-II Division of Medical Department, Key Laboratory of Carcinogenesis and Translational Research \\ (Ministry of Education/Beijing), Peking University Cancer Hospital and Institute, Beijing 100142, P.R. China; \\ ${ }^{2}$ Cardiff China Medical Research Collaborative, Cardiff University School of Medicine, Cardiff CF14 4XW, UK; \\ ${ }^{3}$ Department of Biochemistry and Molecular Biology; ${ }^{4}$ Laboratory of Molecular Oncology, \\ Key Laboratory of Carcinogenesis and Translational Research (Ministry of Education/Beijing), \\ Peking University Cancer Hospital and Institute, Beijing 100142, P.R. China
}

Received March 31, 2017; Accepted October 20, 2017

DOI: $10.3892 /$ ijmm.2017.3224

\begin{abstract}
A characteristic of the epithelial-to-mesenchymal transition in cancer cells is the upregulation of mesenchymal markers. Fibroblast activation protein $\alpha(\mathrm{FAP} \alpha)$ is predominantly expressed by stromal fibroblasts. Previous studies have demonstrated that FAP $\alpha$ is also expressed by certain epithelium-derived cancer cells and is involved in the regulation of certain signaling pathways. One of our previous studies showed that FAP $\alpha$ promoted the proliferation of breast cancer cells via the phosphatidylinositol-3-kinase (PI3K) signaling pathway. In the present study, the A549 adenocarcinoma (AC) and SK-MES-1 squamous cell carcinoma (SCC) lung cancer cell lines were transfected with FAP $\alpha$. The FAP $\alpha$-expressing SK-MES-1 cells exhibited an increased growth rate, whereas the FAP $\alpha$-expressing A549 cells exhibited a similar growth rate, compared with respective empty vector-transfected control cells. Electric cell-substrate impedance
\end{abstract}

Correspondence to: Dr Xiaodong Zhang, VIP-II Division of Medical Department, Key Laboratory of Carcinogenesis and Translational Research (Ministry of Education/Beijing), Peking University Cancer Hospital and Institute, 52 Fucheng Road, Haidian, Beijing 100142, P.R. China

E-mail: zhangxd0829@163.com

Professor Wen G. Jiang, Cardiff China Medical Research Collaborative, Cardiff University School of Medicine, Cardiff CF14 4XW, UK

E-mail: jiangw@cf.ac.uk

Abbreviations: FAP $\alpha$, fibroblast activation protein $\alpha$; $\mathrm{SHH}$, sonic hedgehog; PI3K, phosphatidylinositol-3-kinase

Key words: phosphatidylinositol-3-kinase, fibroblast activation protein $\alpha$, lung cancer, sonic hedgehog sensing (ECIS)-based attachment and wound-healing assays showed that the overexpression of FAP $\alpha$ markedly increased the adhesive and migratory properties of the SK-MES-1 cells but not those of the A549 cells. Additionally, inhibitors of focal adhesion kinase, agonist-induced phospholipase C, neural Wiskott-Aldrich syndrome protein, extracellular signal-regulated kinase, Rho-associated protein kinase, PI3K, and sonic hedgehog $(\mathrm{SHH})$ were used to evaluate the interaction between FAP $\alpha$ and signaling pathways. Only the inhibitors of SHH and PI3K inhibited the increased motility of the FAP $\alpha$-expressing SK-MES-1 cells. Western blot analysis confirmed the activation of PI3K/AKT and SHH/GLI family zinc finger 1 signaling in the FAP $\alpha$-expressing SK-MES-1 cells. These results revealed that FAP $\alpha$ promoted the growth, adhesion and migration of lung SCC cells. In addition, FAP $\alpha$ regulated lung cancer cell function, potentially via the PI3K and SHH pathways. Further investigations are required to examine the role of FAP $\alpha$ in lung AC cells.

\section{Introduction}

The epithelial-to-mesenchymal transition (EMT) is a complicated and critical process in the metastatic spread of cancer cells. The transition of morphology of a cancer cell from an epithelial to a mesenchymal morphology leads to increased migratory and invasive cellular properties. Previous studies have demonstrated that EMT in cancer cells involves the upregulation of mesenchymal markers and the downregulation of epithelial cell markers $(1,2)$. For example, fibroblast specific protein-1 (S100A4) is a prototypical fibroblast marker of EMT in cancer; the expression of S100A4 is associated with cellular motility and the regulation of cell polarization through its effects on the localization of protrusions, which involves interactions with myosin-IIA $(1,3)$. The $\alpha$-smooth muscle actin ( $\alpha$ SMA) protein is expressed by vascular smooth muscle and myoepithelial cells, and is also considered a marker of EMT. There is also evidence that type 3 EMT is associated with $\alpha$ SMA in basal-like breast cancer $(2,4)$. Therefore, the 
function of these molecules expressed by stromal fibroblasts and epithelium-derived cancer cells may be importance in EMT.

Fibroblast activation protein- $\alpha($ FAP $\alpha)$ is an integral membrane serine peptidase. Previous studies have shown that FAP $\alpha$ is expressed primarily in fetal mesenchymal tissues, stromal fibroblasts, wounded tissues and stromal fibroblasts of malignant epithelial tumors (5-7). FAP $\alpha$ expressed by stromal cancer-associated fibroblasts has dipeptidyl peptidase activity $(8,9)$ and collagenolytic activity $(10,11)$. By degrading the extracellular matrix, FAP $\alpha$ promotes the growth and metastasis of cancer cells (12-14). The overexpression of FAP $\alpha$ is also associated with distant metastasis, tumor recurrence and poor survival rates $(15,16)$. In addition, previous studies have shown that FAP $\alpha$ is expressed in certain types of epithelium-derived cancer, including breast (17), gastric (18), esophageal (19), ovarian (20) and colorectal cancer (21).

The functions of cancer cell-expressed FAP $\alpha$ have been investigated in a number of studies and the results from these suggest that FAP $\alpha$ has a non-enzymatic function. Wang et al analyzed the effect of the overexpression of FAP $\alpha$ on the LX-2 human hepatic stellate cell line (22); it was found that the overexpression of FAP $\alpha$ increased the adhesion, migration and invasion of LX-2 cells, and that the proteolytic activity of FAP $\alpha$ was not necessary for these functions (22). Huang et al used two inhibitors, PT-630 and LAF-237, to inhibit the dipeptidyl peptidase activity of FAP $\alpha$ (23), and found that the inhibitors were unable to slow the growth of tumors in severe combined immunodeficient (SCID) mice implanted with FAP $\alpha$-expressing breast cancer WTY-1/6 cells (MDA MB-231 cells transfected with FAP $\alpha$ ) and MDA-MB-435 cells (endogenously express FAP $\alpha$ ). In addition, breast cancer cells expressing a catalytically inactive mutant of FAP $\alpha$ produced tumors, which grew rapidly (23). Wang et al found that the knockdown of FAP $\alpha$ in oral squamous cancer cells suppressed cell proliferation in vitro and inhibited the growth of tumor xenografts in mice in vivo. Notably, suppressing FAP $\alpha$ in oral squamous cancer cells significantly decreased the expression of phosphorylated phosphatidylinositol-3-kinase (PI3K), protein kinase $\mathrm{B}(\mathrm{AKT})$, mitogen-activated protein kinase kinase $1 / 2$ and extracellular signal-regulated kinase (ERK)1/2, and upregulated the expression of phosphatase and tensin homolog (PTEN) (24). In our previous study (25), the overexpression of wild-type FAP $\alpha$ and the enzymatic mutant FAPS624A, in which the serine catalytic triad was disrupted, markedly increased cellular growth and motility in MCF-7 breast cancer cells. These observations were consistent with those for FAP $\alpha$-silenced BT549 breast cancer cells. Western blot analysis also revealed that the overexpression of wild-type FAP $\alpha$ and the enzymatic mutant FAPS624A resulted in the activation of PI3K/AKT and matrix metalloproteinase 2/9 (MMP2/9) (25). These results suggested that FAP $\alpha$ may serve as an oncogene and be involved in the regulation of cell signaling pathways.

Therefore, the present study analyzed the function of FAP $\alpha$ in lung cancer cells in order to examine its non-enzymatic function. It was hypothesized that, as a membrane protein, FAP $\alpha$ may be involved in the regulation of certain signaling pathways and, through this mechanism, exert its effect on lung cancer cells.

\section{Materials and methods}

Materials and cell lines. The SK-MES-1 squamous cell carcinoma (SCC) and A549 adenocarcinoma (AC) human lung cancer cell lines were obtained from the American Type Culture Collection (Manassas, VA, USA). Primary antibodies against human FAP $\alpha$ (cat. no. AF3715) were from R\&D Systems, Inc. (Minneapolis, MN, USA). Primary antibodies against glyceraldehyde 3-phosphate dehydrogenase (GAPDH; cat. no. sc-32233) and an inhibitor (Y-27632; cat. no. sc-3536) of Rho-associated protein kinase (ROCK) were from Santa Cruz Biotechnology, Inc. (Santa Cruz, CA, USA). The focal adhesion kinase (FAK) inhibitor (cat. no. 3414) was from Tocris Bioscience (Bristol, UK). The ERK1/2 inhibitor (FR180204, cat. no. 328007), phospholipase C- $\gamma$ (PLC $\gamma$ ) inhibitor (U73122, cat. no. 662035), neural Wiskott-Aldrich syndrome protein (NWASP) inhibitor (wiskostatin, cat. no. 681525), PI3K inhibitor (wortmannin, cat. no. 681675), c-Jun N-terminal kinase (JNK) inhibitor (SP600125, cat. no. 420119), sonic hedgehog (SHH) inhibitor (cyclopamine, cat. no. 239803) and a negative control of cyclopamine (tomatidine, cat. no. 614350) were from Calbiochem; Merck Millipore (Darmstadt, Germany). Matrigel (reconstituted basement membrane) was purchased from Collaborative Biomedical Products, Inc. (Bedford, MA, USA). Transwell plates equipped with a porous insert ( $8-\mu \mathrm{m}$ pore size) were from BD Biosciences (Franklin Lakes, NJ, USA). Reverse transcription-polymerase chain reaction (RT-PCR) reagents and plasmid extraction kits were from Sigma-Aldrich, Inc. (St. Louis, MO, USA). Antibodies against human PI3K (cat. no. ab22653), AKT (cat. no. ab8805), SHH (cat. no. ab53281), Patched (Ptch; cat. no. ab53715), and GLI family zinc finger 1 (Gli1; cat. no. ab151796) were from Abcam PLC (Cambridge, UK).

Construction of FAP $\alpha$-expressing lung cancer cell lines. The cDNA of FAP $\alpha$ (NM_004460) was inserted into a pEF6/V5-His vector (Invitrogen; Thermo Fisher Scientific, Inc., Waltham, MA, USA) and maintained at $-80^{\circ} \mathrm{C}$ in the Laboratory of Carcinogenesis and Translational Research, Peking University Cancer Hospital and Institute, as described in our previous study (25). The SK-MES-1 and A549 lung cancer cells were cultured in DMEM/F12 media at $37^{\circ} \mathrm{C}$ with $5 \% \mathrm{CO}_{2}$. The cells were transfected with the pEF6/V5-FAP $\alpha$ plasmid by electroporation. Following the selection of transfected cells with blasticidin $\mathrm{S}(5 \mu \mathrm{g} / \mathrm{ml})$ and verification via RT-PCR analysis (RT-reaction: annealing at $65^{\circ} \mathrm{C}$ for $5 \mathrm{~min}$, extension at $55^{\circ} \mathrm{C}$ for $20 \mathrm{~min}$, inactivation at $75^{\circ} \mathrm{C}$ for $15 \mathrm{~min}$. PCR reaction: initial denaturation at $94^{\circ} \mathrm{C}$ for $5 \mathrm{~min}$; then denaturation at $94^{\circ} \mathrm{C}$ for $30 \mathrm{sec}$, annealing at $55^{\circ} \mathrm{C}$ for $30 \mathrm{sec}$, extension at $72^{\circ} \mathrm{C}$ for $1 \mathrm{~min}$ in each cycle for totally 25 cycles, followed by a final extension of $10 \mathrm{~min}$ at $72^{\circ} \mathrm{C}$ ), the following stably transfected cells were established: FAP $\alpha$-overexpressing cells (SK-MES-1exp and A549exp), plasmid vector-transfected control cells (SK-MES-1pef and A549pef), and wild-type cells (SK-MES-1wt and A549wt). The transfected cells were continually cultured in a maintenance medium, which contained $0.5 \mu \mathrm{g} / \mathrm{ml}$ blasticidin $\mathrm{S}$.

In vitro growth, adhesion and invasion assays. For the growth assay, the cells were plated into a 96-well plate at a density of 2,000 cells/well $(n=12)$ followed by a period of incubation. 
The cells were either fixed in $10 \%$ formaldehyde on the day of plating or 3 days later. Crystal violet $(0.5 \% \mathrm{w} / \mathrm{v})$ was used to stain the cells. Subsequently, the crystal violet was dissolved with $10 \%(\mathrm{v} / \mathrm{v})$ acetic acid and the absorbance of cells, which represented the cell number, was determined at a wavelength of $540 \mathrm{~nm}$.

For the adhesion assay, a 96-well plate was pre-coated with $5 \mu \mathrm{g}$ of Matrigel and left to dry overnight. Following rehydration with serum-free media, 1,500 cells were seeded into each well $(n=6)$. Following incubation for $60 \mathrm{~min}$, the non-adherent cells were washed off using BSS buffer. The remaining cells were fixed with $4 \%$ formalin and stained with $0.5 \%$ crystal violet. The numbers of adherent cells were then counted under an Olympus CX31 microscope.

For the invasion assay, Transwell inserts with a $8-\mu \mathrm{m}$ pore size were coated with $50 \mu \mathrm{g}$ of Matrigel and air-dried. Following rehydration with serum-free media, the cells were seeded at a density of 30,000 cells/insert $(n=5)$. After 3 days of incubation at $37^{\circ} \mathrm{C}$ with $5 \% \mathrm{CO}_{2}$, the cells, which had migrated through the matrix and adhered to the other side of the insert were fixed in $4 \%$ formalin, stained with $0.5 \%$ (w/v) crystal violet, and counted under a microscope.

Electric cell-substrate impedance sensing (ECIS)-based cell attachment and migration assay. An ECIS instrument (Applied BioPhysics, Inc., Troy, NY, USA) was used to record the adhesive and migratory abilities of the cells, which were determined from the changes in the impedance of cells. $96 \mathrm{~W} 1 \mathrm{E}$ arrays were incubated with complete medium for $1 \mathrm{~h}$, following which 50,000 lung cancer cells were seeded into each well $(n=16)$. Electrical changes were continuously monitored for up to $24 \mathrm{~h}$, with electrical wounding performed at $6 \mathrm{~h}$. Multiple frequencies $(1,000,2,000,4,000$ and 8,000 Hz) were used to assess the nature of changes in resistance.

Inhibition of signaling pathways with inhibitors. In order to examine the potential crosstalk between FAP $\alpha$ and adhesion and migration-associated signaling pathways, inhibitors of FAK, ERK1/2, ROCK, PLC $\gamma$, JNK, NWASP, PI3K and SHH were used in an ECIS-based cell function assay. A total of 50,000 cells were suspended in $200 \mu$ l DMEM with inhibitors of FAK, ERK1/2, ROCK, PLC $\gamma$, NWASP, JNK, PI3K and $\mathrm{SHH}$, respectively, to a final concentration of $100 \mathrm{nM}$. Changes in electrical resistance under multiple frequencies $(1,000$, 2,000, 4,000 and $8,000 \mathrm{~Hz}$ ) were continuously monitored for up to $24 \mathrm{~h}$, with electrical wounding performed at $6 \mathrm{~h}$ in the ECIS-based wounding assay $(n=8)$.

Western blot analysis. Western blot analysis was performed to detect the expression of FAP $\alpha$ and other downstream signaling pathway molecules in the transfected SK-MES-1 lung cancer cells and control cells. The confluent cells were pelleted and then lysed in lysis buffer containing $2.4 \mathrm{mg} / \mathrm{ml}$ Tris, $4.4 \mathrm{mg} / \mathrm{ml}$ $\mathrm{NaCl}, 5 \mathrm{mg} / \mathrm{ml}$ sodium deoxycholate, $20 \mu \mathrm{g} / \mathrm{ml}$ sodium azide, $1.5 \%$ Triton X-100, $100 \mu \mathrm{g} / \mathrm{ml}$ phenylmethylsulfonyl fluoride, $1 \mu \mathrm{g} / \mathrm{ml}$ leupeptin and $1 \mu \mathrm{g} / \mathrm{ml}$ aprotinin, for $45 \mathrm{~min}$ at $4^{\circ} \mathrm{C}$. Following lysis and centrifugation at $10,000 \mathrm{x}$ g for $15 \mathrm{~min}$ at $4^{\circ} \mathrm{C}$, the isolated proteins were diluted in sample buffer and the concentration of each sample was measured using an improved Lowry assay (DC protein assay kit; Bio-Rad Laboratories,
Inc., Hercules, CA, USA). The samples were adjusted to equal concentrations $(2 \mathrm{mg} / \mathrm{ml})$ with sample buffer and then boiled at $100^{\circ} \mathrm{C}$ for $5 \mathrm{~min}$ prior to separation on a $10 \%$ polyacrylamide gel. Following electrophoresis, the separated protein samples were transferred onto polyvinylidenedifluoride membranes (EMD Millipore, Billerica, MA, USA) and incubated at $4^{\circ} \mathrm{C}$ overnight with primary antibodies against FAP $\alpha$, PI3K, AKT, SHH, Ptch, Gli1 and GAPDH at dilution of 1:200. The membranes were then incubated with specific peroxidase-conjugated secondary antibodies (cat. nos. sc-2357 or sc-2031; Santa Cruz Biotechnology, Inc.) at a dilution of 1:1,000 for $40 \mathrm{~min}$ at room temperature. The protein signals were detected using an enhanced chemiluminescence system (Pierce; Thermo Fisher Scientific, Inc.).

Statistical analysis. All results are expressed as the mean \pm standard error of the mean. One-way analysis of variance and an independent samples t-test were used to evaluate the differences between groups in all the assays performed, with the resultant P-values representing two-sided tests of statistical significance. All statistical analyses were performed using SPSS 17.0 software (SPSS, Inc., Chicago, IL, USA). P $<0.05$ was considered to indicate a statistically significant difference.

\section{Results}

Expression of FAP $\alpha$ in lung cancer cells. To identify the oncogenic function of FAP $\alpha$ in lung cancer cells, FAP $\alpha$ was transfected into the FAP $\alpha$-null SK-MES-1 and A549 lung cancer cell lines. RT-PCR and western blot analyses were used to confirm the expression of FAP $\alpha$ in the transfected cells. The overexpression of FAP $\alpha$ in the SK-MES-1 and A549 cells was successfully established (Fig. 1).

Overexpression of FAP $\alpha$ promotes the growth of SK-MES-1 cells. The present study first examined the effect of the overexpression of FAP $\alpha$ on cellular growth in vitro. Compared with the SK-MES-1wt cells, the overexpression of FAP $\alpha$ significantly increased the growth rate of the SK-MES-1exp cells at 3 days post-seeding $(n=12 ; 575.5 \pm 171.9$ vs. $793.6 \pm 140.1 \%$, respectively; $\mathrm{P}=0.003$ ), which was also significantly higher, compared with that of the SK-MES-1pef cells (611.6 \pm 181.8 ; $\mathrm{P}=0.012)$. By contrast, the growth rate of the A549exp cells $(357.5 \pm 74.2 \%)$ was similar to the growth rates of the A549wt cells $(370.6 \pm 83.2 ; \mathrm{n}=12 ; \mathrm{P}=0.686)$ and A549pef cells (357.8 $\pm 57.4 \%, \mathrm{P}=0.989)$ at 3 days post-seeding (Fig. 2).

Overexpression of FAP $\alpha$ increases the attachment of SK-MES-1 cells to basement membrane proteins. In a matrix gel-based adhesion assay, compared with the control cells, FAP $\alpha$-expressing SK-MES-1exp and A549exp cells showed increased adhesion to the matrix gel $1 \mathrm{~h}$ following seeding; however, no statistically significant differences were found between the groups of SK-MES cells $(n=6$; SK-MES-1wt vs. SK-MES-1exp, $25 \pm 14.4$ vs. $37.3 \pm 19.9 ; \mathrm{P}=0.246$; SK-MES-1pef vs. SK-MES-1exp, $31.7 \pm 10.3$ vs. $37.3 \pm 19.9 ; \mathrm{P}=0.549$ ) or $\mathrm{A} 549$ cells $(\mathrm{A} 549 \mathrm{wt}$ vs. A549exp, $17.7 \pm 15.7$ vs. $24.5 \pm 12.3 ; \mathrm{P}=0.422$; A549pef vs. A549exp, $14.5 \pm 14.4$ vs. $24.5 \pm 12.3, \mathrm{P}=0.225$ ) (Fig. $3 \mathrm{~A}$ and $\mathrm{B}$ ). To confirm the results of the matrix gel-based adhesion assay, an ECIS-based cell function assay was performed as a more 

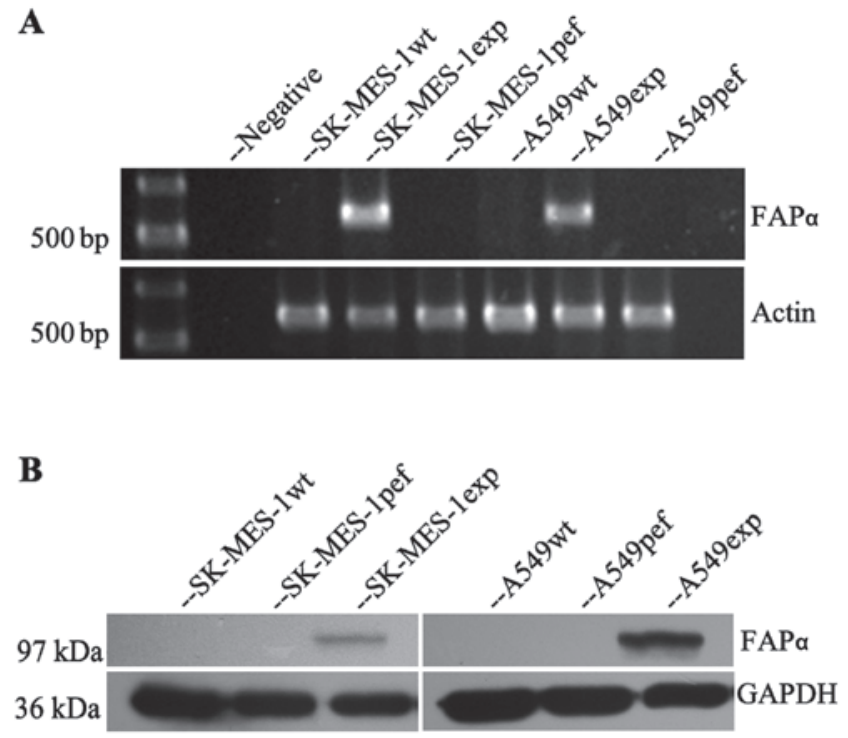

Figure 1. Determination of the overexpression efficiency of FAP $\alpha$ in lung cancer cells. (A) mRNA transcription of FAP $\alpha$ was negative in SK-MES-1wt and A549wt lung cancer cells and SK-MES-1pef and A549pef cells, but positive in SK-MES-1exp and A549exp cells. (B) Western blot analysis confirmed the protein expression of FAP $\alpha$ in SK-MES-1exp and A549exp cells. FAP $\alpha$, fibroblast activation protein $\alpha$; wt, wild-type cells; pef, vector-transfected control cells; exp, FAP $\alpha$-expressing cells.

sensitive and accurate assessment. In the first phase post-cell seeding, increased impedance reflects the ability of cells to adhere to the substrate; the faster the impedance increases, the higher the rate of cell attachment to the substrate. In the present study, compared with the SK-MES-1wt and SK-MES-1pef control cells, the FAP $\alpha$-expressing SK-MES-lexp cells showed increased attachment at $1 \mathrm{~h}$ post-seeding $(\mathrm{n}=12$; SK-MES-1wt vs. SK-MES-1exp, $343.1 \pm 135.3$ vs. $948.3 \pm 442.7$ ohms; $\mathrm{P}=0.001$; SK-MES-1pef vs. SK-MES-1exp, $566.3 \pm 321.0$ vs. $948.3 \pm 442.7$ ohms; $\mathrm{P}=0.037)$. However, in the A549 cells, the vector-transfected A549pef cells showed the highest attachment ability, whereas the A549exp cells and A549wt cells exhibited similar attachment abilities ( $\mathrm{n}=12$; A549wt vs. A549exp, 234.1 \pm 149.4 vs. 292.2 \pm 191.0 ohms; $\mathrm{P}=0.416 ;$ A549pef vs. A549exp, 497.7 \pm 184.5 vs. 292.2 \pm 191.0 ohms; $\mathrm{P}=0.014$ ) (Fig. 3C-E).

Overexpression of FAP $\alpha$ does not promote the invasion of lung cancer cells. To analyze the effect of the overexpression of FAP $\alpha$ on the invasion of lung cancer cells, the present study performed an in vitro matrix gel-based invasion assay. Although FAP $\alpha$ has dipeptidyl peptidase and collagenolytic activities, the results showed that the overexpression of FAP $\alpha$ did not increase the invasive ability of either SK-MES-1 or A549 cells. By contrast, the number of invaded cells in the FAP $\alpha$-expressing SK-MES-1 cell group on day 3 was lower, compared with that in the wildtype and vector-transfected control cell groups; however, no significant differences were observed between the groups $(\mathrm{n}=5$; SK-MES-1wt vs. SK-MES-1exp, $184.2 \pm 77.8$ vs. $110.4 \pm 11.4$; $\mathrm{P}=0.138$; SK-MES-1pef vs. SK-MES-1exp, $126.0 \pm 13.2$ vs. $110.4 \pm 11.4 ; \mathrm{P}=0.081)$. In the A549 cells, the number of invaded cells in the FAP $\alpha$-expressing A549exp cell group on day 3 was similar to that in the A549wt cell group $(\mathrm{n}=5 ; 37.8 \pm 15.4$ vs. $42.0 \pm 6.5$, respectively; $\mathrm{P}=0.59)$, but less than that in the A549pef group ( $88.8 \pm 17.9$ vs. $37.8 \pm 15.4 ; \mathrm{P}=0.001)$ (Fig. 4).

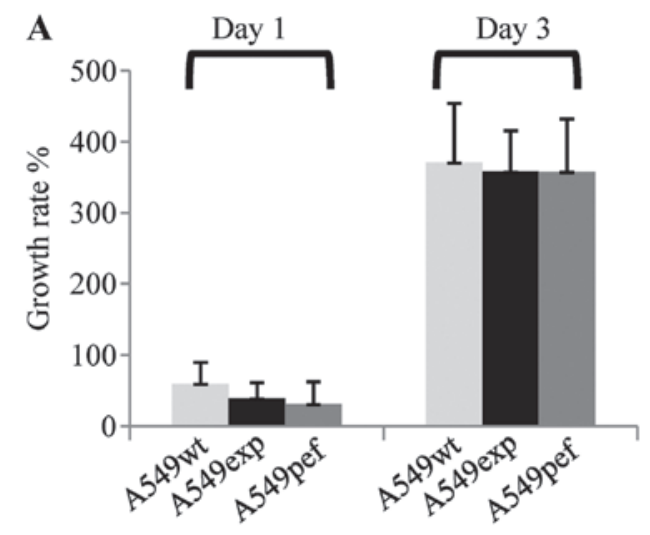

B

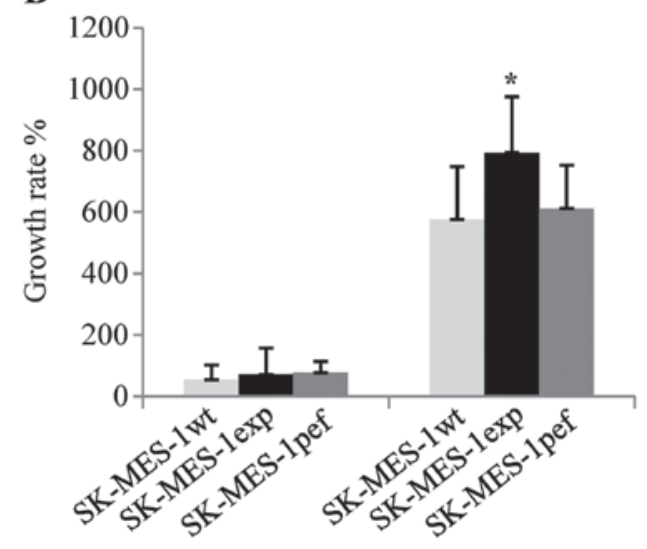

Figure 2. Overexpression of FAP $\alpha$ promotes the growth of SK-MES-1 cells Overexpression of FAP $\alpha$ had no significant effect on the growth rate of (A) A549 cells but significantly increased the growth rate of (B) SK-MES-1 cells at 3 days post-seeding $(n=12)$. ${ }^{*} \mathrm{P}<0.05$ vs. SK-MES-1wt and SK-MES-1pef. FAP $\alpha$, fibroblast activation protein $\alpha$; exp, FAP $\alpha$-expressing cells; pef, vector-transfected control cells; wt, wild-type cells.

Overexpression of FAP increases the migration of SK-MES-1 cells. To investigate the effect of FAP $\alpha$ on the migration of lung cancer cells, the more accurate ECIS-based wounding assay was used rather than a physical scratch-wound assay. In the ECIS method, the wound is created in the confluent cell monolayer using a high voltage shock, and the faster the increase in impedance following wounding, the higher the rate of cellular migration into the wound. As an additional measure of accuracy, the change of impedance is recorded automatically rather than using a manual measurement. In the present study, the overexpression of FAP $\alpha$ significantly elevated the migration ability of SK-MES-1 cells $4 \mathrm{~h}$ post-wounding ( $\mathrm{n}=16$; SK-MES-1wt vs. SK-MES-1exp, $200.0 \pm 173.2$ vs. $394.8 \pm 254.5$ ohms; $\mathrm{P}=0.001$; SK-MES-1pef vs. SK-MES-1exp, $228.0 \pm 282.6$ vs. $394.8 \pm 254.5$ ohms; $\mathrm{P}=0.017$ ). However, the overexpression of FAP $\alpha$ in A549 cells had no effect on cell migration rate when compared with control cells $4 \mathrm{~h}$ post-wounding ( $\mathrm{n}=16$; A549wt vs. A549exp: $578.8 \pm 215.7 \mathrm{vs.}$ $610.2 \pm 182.7$ ohms; $\mathrm{P}=0.66$; A549pef vs. A549exp, 580.2 \pm 221.8 vs. $610.2 \pm 182.7$ ohms; $\mathrm{P}=0.68$ ) (Fig. 5).

Inhibitors of SHH and PI3K inhibit the increases in cell attachment and migration induced by the overexpression of FAP $\alpha$. To examine the potential interaction of FAP $\alpha$ with signaling pathways potentially responsible for the increased adhesive and migratory properties of SK-MES-1 lung cancer cells, a panel of small-molecule inhibitors of a number of signaling pathways 
A

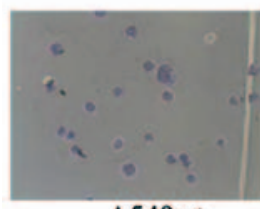

A549wt

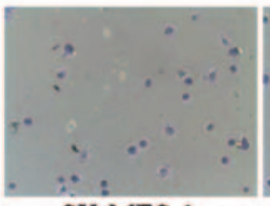

SK-MES-1wt

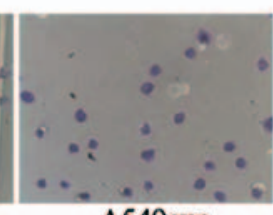

A549exp

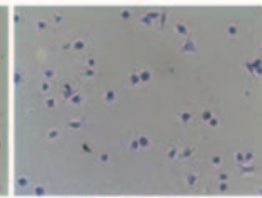

SK-MES-lexp

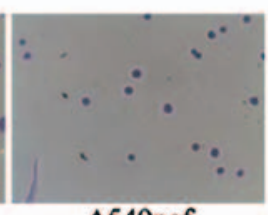

A549pef

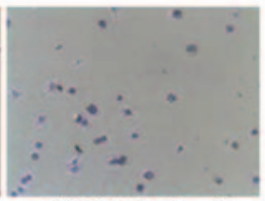

SK-MES-Ipef

B

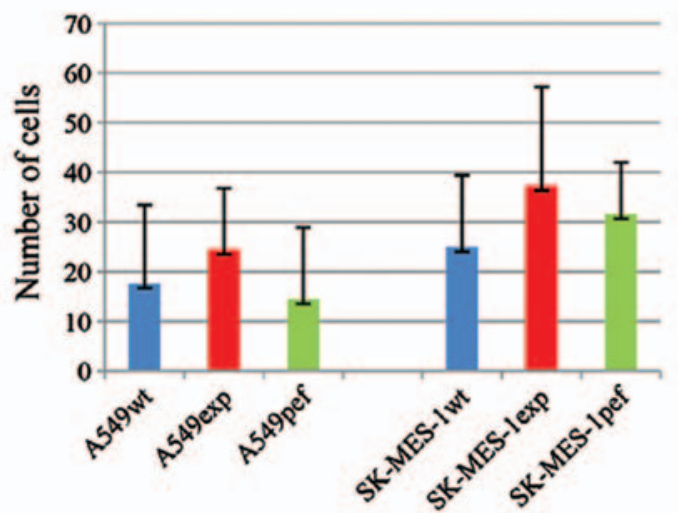

C
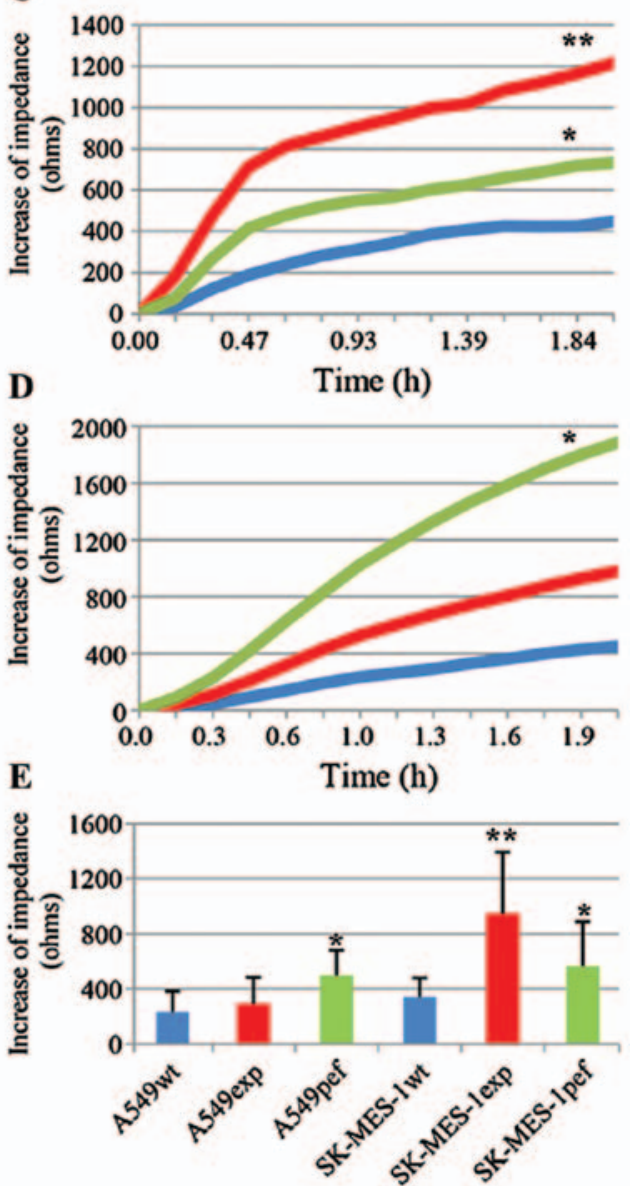

Figure 3. Overexpression of FAP $\alpha$ increases the attachment of SK-MES-1 cells to the basement substrate. (A and B) Results of the cell matrix adhesion assay showed that the overexpression of FAP $\alpha$ enhanced the adhesive properties of SK-MES-1 and A549 cells, but not significantly (magnification, x200). An ECIS-based attachment assay confirmed the increased attachment ability only in (C) SK-MES-1 cells, but not in (D) A549 cells over time ( $\mathrm{n}=12$ ). (E) The increased impedance of lung cancer cells in attachment phase of ECIS assay at $1 \mathrm{~h}$ after seeding. *P<0.05, SK-MES-1pef vs. SK-MES-1exp and A549pef vs. A549exp; ${ }^{* *} \mathrm{P}<0.01$, SK-MES-1exp vs. SK-MES-1wt. FAP $\alpha$, fibroblast activation protein $\alpha$; exp, FAP $\alpha$-expressing cells; pef, vector-transfected control cells; wt, wild-type cells; ohms, unit of electrical impedance.

were screened, including ROCK, FAK, NWASP, ERK1/2, PLC $\gamma$, JNK, PI3K and SHH. Using an ECIS assay ( $\mathrm{n}=8$ for each experiment), the effects of these inhibitors on cell attachment and migration were assessed. Compared with the SK-MES-1pef cells, only the inhibitors of SHH and PI3K significantly inhibited the increased the cell attachment and motility (Fig. 6A-D) of the FAP $\alpha$-expressing SK-MES-1exp cells.

In the earlier cell function assay, the overexpression of FAP $\alpha$ in A549 cells failed to increase cellular adhesion and migration. The present study subsequently analyzed the effects of the above inhibitors on the attachment and motility of A549 cells. The results showed that there was minimal difference in the attachment rate of the A549exp cells following treatment with the inhibitors; only the inhibitor of FAK stimulated the migration of A549exp cells, with the other inhibitors having no marked effect on cell migration (Fig. 6E-H).

FAP $\alpha$ regulates cellular functions through the PI3K/AKT and $S H H / G l i 1$ pathways. To determine whether the effects of FAP $\alpha$ on the cellular functions of SK-MES-1 cells were associated with the PI3K/AKT and SHH pathways, western blot analysis was performed to detect the protein expression levels of total PI3K, AKT, SHH, Ptch and Gli1. As shown in Fig. 7, compared with the control cells, the protein levels of AKT and PI3K were markedly increased in the SK-MES-1exp cells. The protein levels of SHH and Gli1 were also increased in the SK-MES-1exp cells. By contrast, the levels of Ptch were similar in the SK-MES-1exp and control cells.

\section{Discussion}

FAP $\alpha$ is an integral membrane serine peptidase. Preliminary studies have shown that FAP $\alpha$ is expressed primarily in fetal mesenchymal tissues, stromal fibroblasts, wounded tissues, and stromal fibroblasts of malignant epithelial tumors (5-7). There is increasing evidence that the expression of FAP $\alpha$ is not confined to stromal fibroblasts but is also present in various types of epithelium-derived cancer cells (17-20). Therefore, the role of FAP $\alpha$ in cancer cells may differ from that in fibroblasts. Goodman et al found that the suppression of FAP $\alpha$ in MDA-MB-435 and MDA-MB-436 human breast cancer cell lines, which normally express FAP $\alpha$, rendered these cells sensitive to serum starvation (26). Cheng et al reported that mice inoculated with FAP $\alpha$-transfected HEK293 cells were two to four times more likely to develop tumors, compared with those inoculated with FAP $\alpha$-null control cells (27). Additionally, in an 

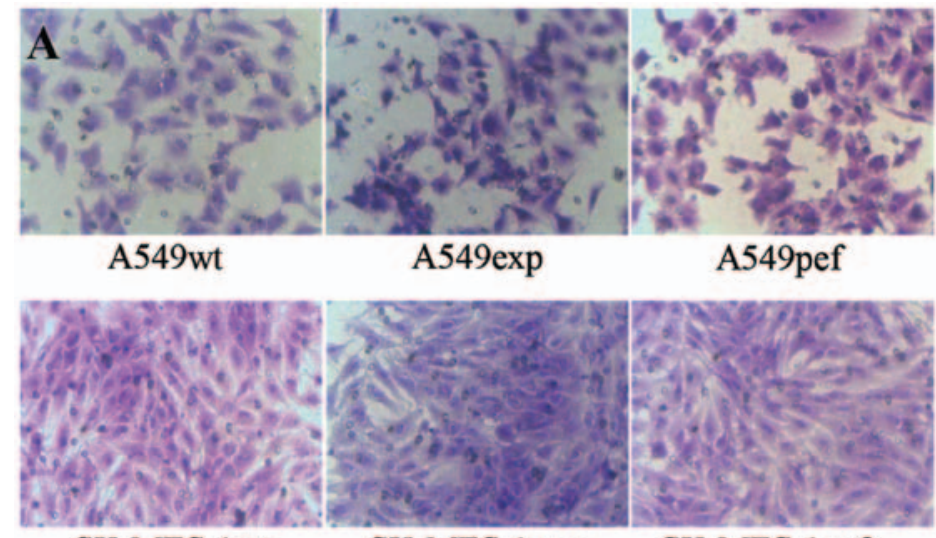

\section{SK-MES-1wt SK-MES-1exp SK-MES-1pef}

B

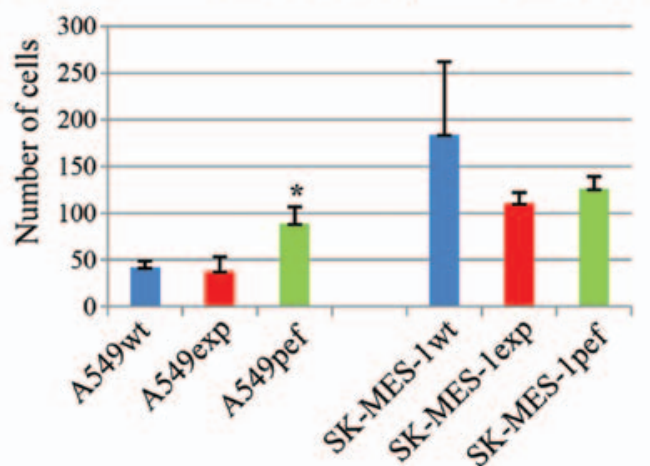

Figure 4. Overexpression of FAP $\alpha$ has no significant effect on the invasive ability of lung cancer cells. (A) Matrix gel-based invasion assay with lung cancer cells was performed 3 days post-seeding (magnification, $\mathrm{x} 400$ ). (B) Numbers of invaded cells in the SK-MES-lexp cell group were lower, compared with those in the SK-MES-1wt and SK-MES-1pef cell groups, but the differences were not significant. The number of invaded cells in the A549exp cell group was similar to that in the A549wt cell group, but was lower, compared with that in the A549pef cell group ( $\mathrm{n}=5)$. ${ }^{*} \mathrm{P}<0.01$ vs. A549exp. FAP $\alpha$, fibroblast activation protein- $\alpha$; exp, FAP $\alpha$-expressing cells; pef, vector-transfected control cells; wt, wild-type cells.

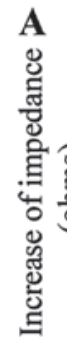

C

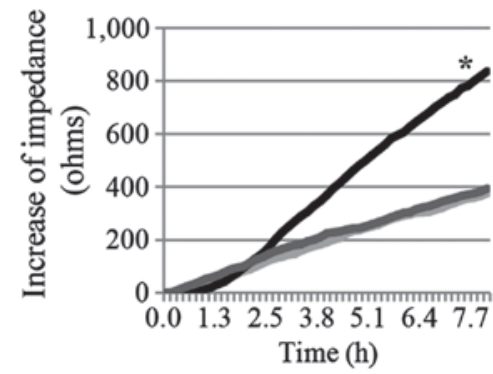

B

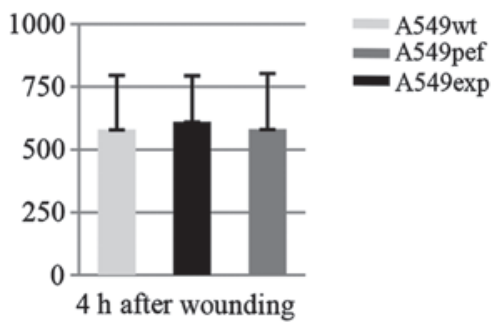

D

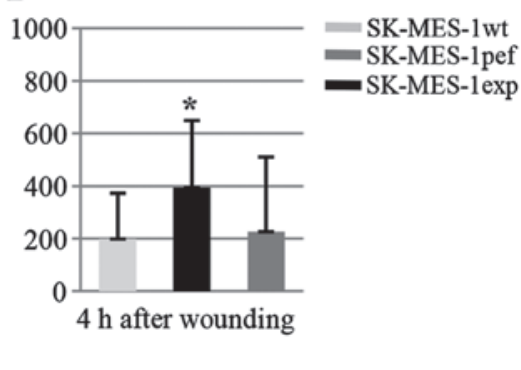

Figure 5. Overexpression of FAP $\alpha$ significantly increases the migratory ability of SK-MES-1 cells. (A and B) An ECIS-based wounding assay showed that the overexpression of FAP $\alpha$ in A549 cells had no effect on cell migration rate, compared with the control cells $(\mathrm{n}=16)$. (C and D) Overexpression of FAP $\alpha$ markedly elevated the migratory capacity of the SK-MES-1 cells at $4 \mathrm{~h}$ post-wounding $(\mathrm{n}=16)$. ${ }^{\mathrm{P}}<0.05$ SK-MES-1pef vs. SK-MES-1exp; SK-MES-1wt vs. SK-MES-1exp. FAP $\alpha$, fibroblast activation protein- $\alpha$; exp, FAP $\alpha$-expressing cells; pef, vector-transfected control cells; wt, wild-type cells; ohms, unit of electrical impedance.

in vivo mouse model, FAP $\alpha$-expressing MDA-MB-231 breast cancer cells grew more rapidly than control cells (28). Previous studies have confirmed these early findings and suggested that
FAP $\alpha$ expressed by cancer cells may serve as an oncogene. For example, it was found that FAP $\alpha$ promoted ovarian cancer cell proliferation, drug resistance, invasiveness and migration 

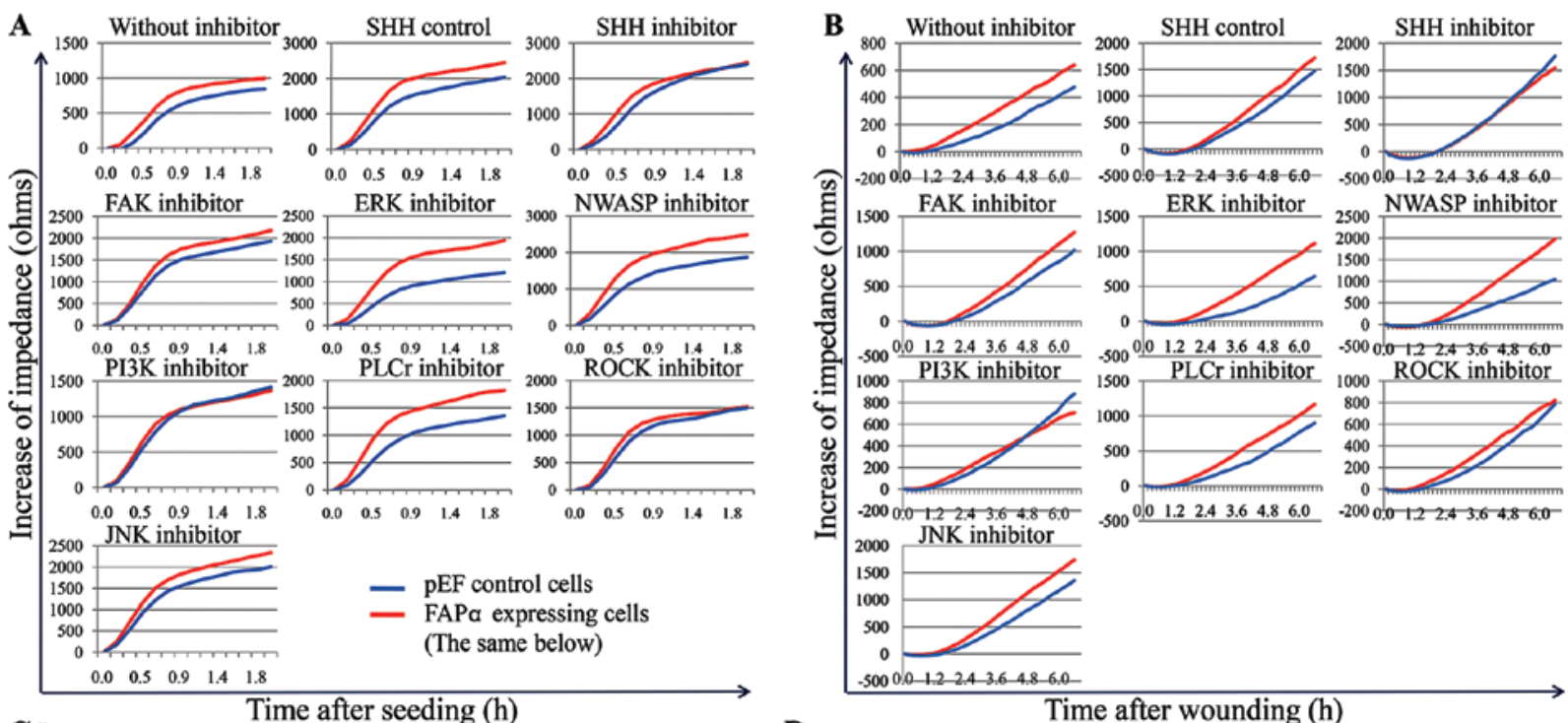

$-2000.0-1.2-2.4-3.6-4.8-6.0$

$5000.0-1.2-2.4-3.6-4.8-6.0$

$5000.0-12-2.4-3.6-4.8-6.0$
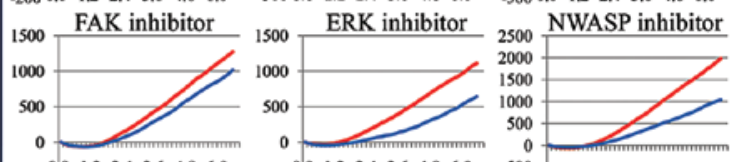

$0.01 .22 .43 .64 .86 .0 \quad 0.01 .22 .43 .64 .86 .0$

-500 PI3K inhibitor

1000 PI3K inhibitor

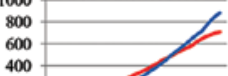

600
400
200
0

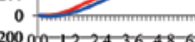

$-200000-1.2-2.4-3.6-4.8-6.0$

2000 JNK inhibitor

1500 PLCr inhibitor

$-5000.0-1.2-2.4-3.6-4.8-6.0$

000 ROCK inhibitor

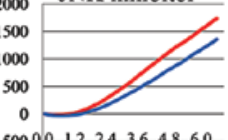

$5000.0-1.2-2.4-3.6-4.8-6.0$

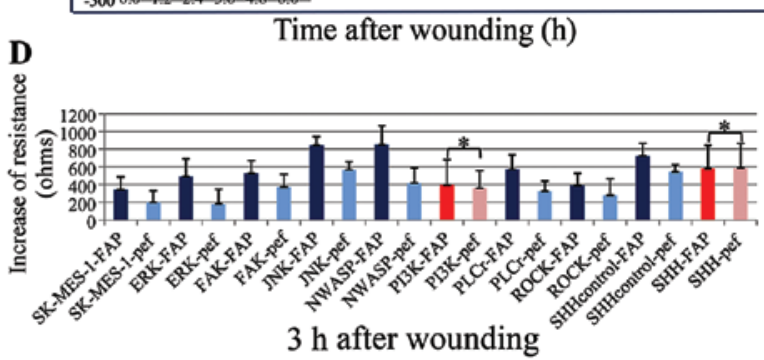

$1.5 \mathrm{~h}$ after seeding

\section{$3 \mathrm{~h}$ after wounding}
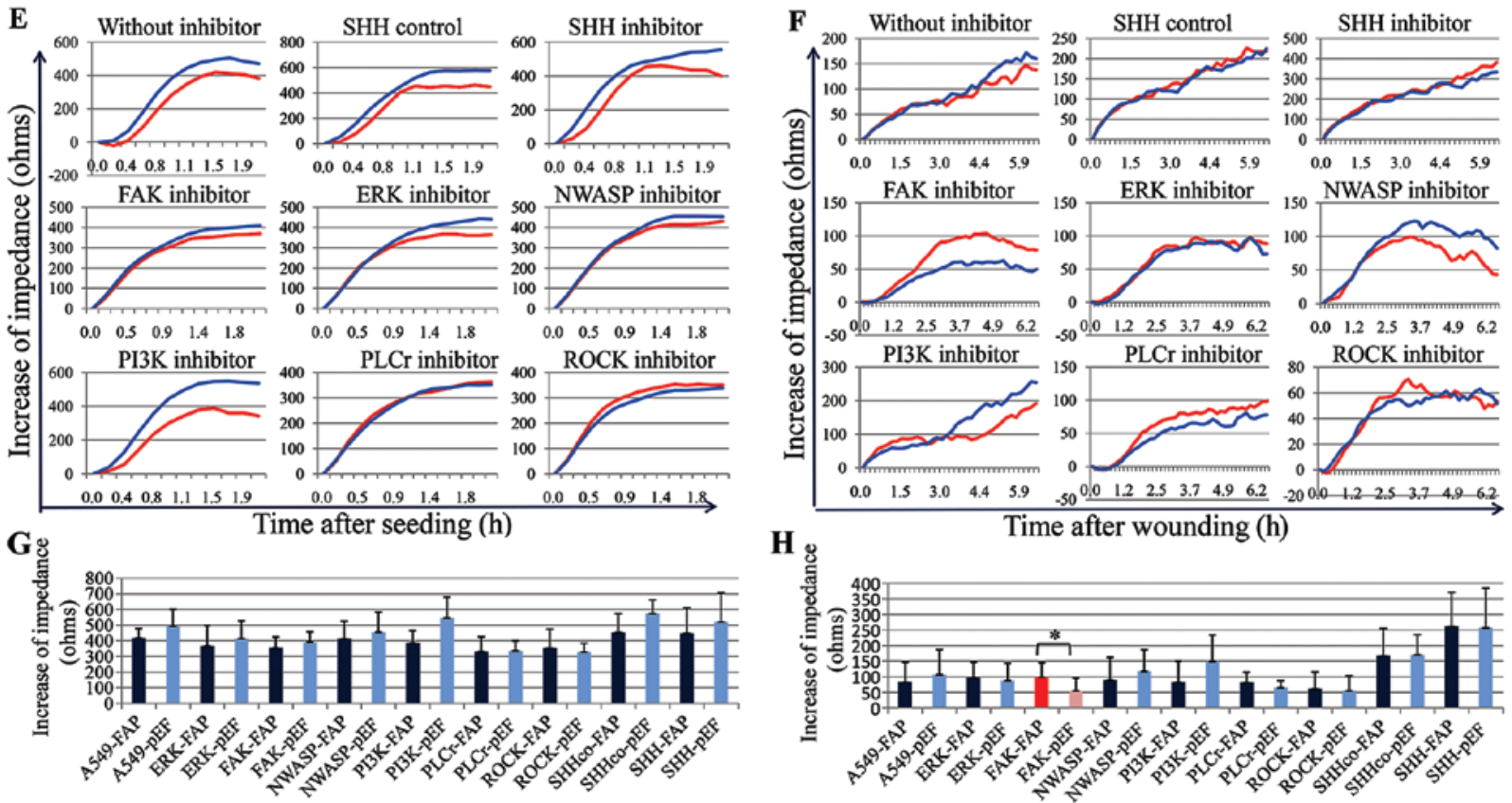

$1.5 \mathrm{~h}$ after seeding

\section{$3 \mathrm{~h}$ after wounding}

Figure 6. Effects of signaling pathway inhibitors on the motility of SK-MES-1 lung cancer cells. A panel of small inhibitors linked to cell motility was screened and only the PI3K and SHH inhibitors had substantial effects. ECIS was used to assess the effects of these inhibitors on the attachment and migration abilities of (A-D) SK-MES-1 cells and (E-H) A549 cells. The PI3K and SHH inhibitors inhibited the increased (A and C) attachment and (B and D) migration of SK-MES-1 cells induced by the overexpression of FAP $\alpha$. In A549 cells, none of the screened inhibitors affected (E and G) cell attachment, and only FAK inhibitor appeared to stimulate $(\mathrm{F}$ and $\mathrm{H})$ cell migration, with the other inhibitors having no marked effect on cell migration. "P $>0.05$. The difference between SK-MES-1exp and SK-MES-1pef cells in the absence of inhibitors was significant $(\mathrm{P}<0.05)$, but addition of inhibitors eliminated the difference. FAP $\alpha$, fibroblast activation protein $\alpha$; FAP, FAP $\alpha$-expressing cells; pEF, vector-transfected control cells; ohms, unit of electrical impedance; SHH, sonic hedgehog; SHHco, control of the SHH inhibitor cyclopamine; FAK, focal adhesion kinase; ERK, extracellular signal-regulated kinase; NWASP, neural Wiskott-Aldrich syndrome protein; PI3K, phosphatidylinositol 3-kinase; PLC $\gamma$, phospholipase C- $\gamma$; ROCK, Rho-associated protein kinase; JNK, c-Jun N-terminal kinase.

in vitro, and that the silencing of FAP $\alpha$ in SKOV3 cells significantly reduced tumor growth in a xenograft mouse model (29). Our previous study (25) showed that the overexpression of
FAP $\alpha$ markedly increased the growth, adhesion, invasion and migration abilities of MCF-7 breast cancer cells, whereas the knockdown of FAP $\alpha$ in BT549 breast cancer cells decreased 


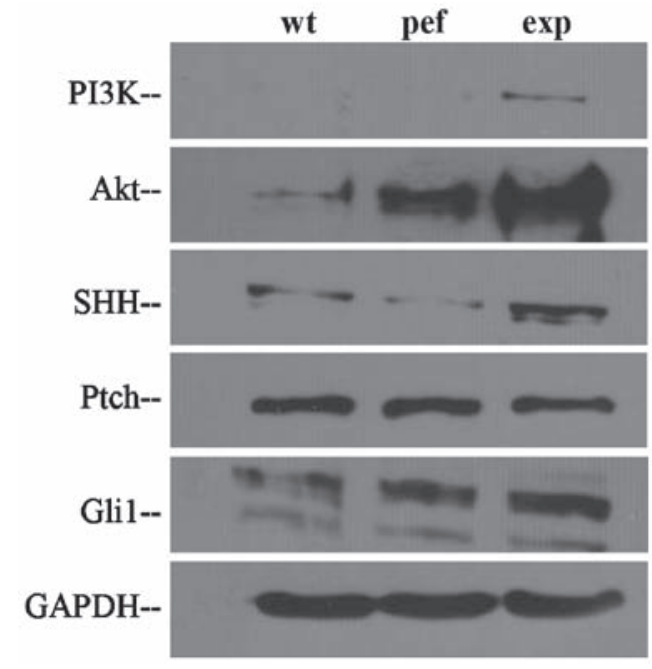

Figure 7. Detection of FAP $\alpha$-mediated effects on the PI3K/AKT and SHH pathways in SK-MES-1 lung cancer cells using western blot analysis. Compared with control cells, the expression levels of AKT, PI3K, SHH and Gli1 were markedly increased in the SK-MES-1exp cells. No marked change in expression of Ptch was observed in the SK-MES-1exp cells. FAP $\alpha$, fibroblast activation protein $\alpha$; exp, FAP $\alpha$-expressing cells; pef, vector-transfected control cells; wt, wild-type cells; PI3K, phosphatidylinositol-3-kinase; AKT, protein kinase B; SHH, sonic hedgehog; Ptch, Patched; GAPDH, glyceraldehyde 3-phosphate dehydrogenase.

these abilities. In the present study, the overexpression of FAP $\alpha$ markedly increased the growth, adhesion and migration of SK-MES-1 lung cancer cells. Therefore, these results suggested that the FAP $\alpha$ expressed by epithelial cancer cells had an oncogenic function. However, the overexpression of FAP $\alpha$ failed to promote the growth and motility of A549 cells in the present study. One reason for this may be the difference in cell type between SK-MES-1 (SCC) and A549 (AC) cells. However, Du et al reported that FAP $\alpha$ was expressed in AC specimens from patients with lung cancer (30). Therefore, the role of FAP $\alpha$ in lung AC cells requires clarification in further investigations.

The detailed mechanisms by which FAP $\alpha$ in epithelial cancer cells promote cell growth and motility remains to be fully elucidated. However, studies have revealed that FAP $\alpha$ has important non-enzymatic functions, which enable it to regulate the proliferation and spread of cancer cells $(22,23,25)$. Huang et al found that inhibitors of FAP $\alpha$ did not decrease the growth of FAP $\alpha$-expressing breast cancer cells, and that breast cancer cells expressing a catalytically inactive mutant of FAP $\alpha$ produced tumors rapidly in a SCID mouse model (23). Our previous study also showed that an enzymatic mutant of FAP, in which the serine catalytic triad was disrupted, markedly increased cellular growth and motility in MCF-7 breast cancer cells (25). Taken together with previous results, it appears that the tumorigenicity of FAP $\alpha$ expressed by cancer cells is not dependent on its enzymatic activity. There is also increasing evidence that FAP $\alpha$ is involved in the regulation of signaling pathways. In oral SCC cells, it has been reported that the knockdown of FAP $\alpha$ inactivated the PTEN/PI3K/AKT and Ras-ERK signaling pathways, resulting in the suppression of oral SCC cell proliferation, migration and invasion (24). In ovarian cancer cells, FAP $\alpha$ in combination with integrin $\alpha 3 \beta 1$ and the UPAR signaling complex mediate cellular migration via the small GTPase Rac1 pathway (31). Our previous study showed that the FAP $\alpha$-mediated promotion of cell growth and motility in breast cancer cells was accompanied by the upregulation of MMP2/9, phosphorylated-PI3K and phosphorylated-AKT (25). In addition, FAP $\alpha$ expressed by stromal cells has been reported to be involved in the regulation of signaling pathways, including RhoA (32), $\beta$-catenin (33) and fibroblast growth factor 1 (FGF1)/FGF receptor 3 (34). In the present study, the potential interactions of FAP $\alpha$ with a number of signaling pathways were analyzed, including FAK, ERK1/2, ROCK, PLC $\gamma$, JNK, NWASP, PI3K and SHH. The results showed that, for SK-MES-1 cells, treatment with PI3K or SHH inhibitors significantly inhibited the increase in cellular motility induced by the overexpression of FAP $\alpha$. The results of the western blot analysis showed that the overexpression of FAP $\alpha$ was accompanied by increases in the protein expression levels of PI3K, AKT, SHH and Gli1, which indicated activation of the PI3K-AKT and SHH-Glil pathways.

There remains no direct evidence to show that FAP $\alpha$ is involved in the EMT process of cancer cells. However, Du et al analyzed the expression of transforming growth factor- $\beta$ (TGF- $\beta$ ), Twist and FAP $\alpha$ in lung cancer tissues, and found that these three molecules were expressed at various levels in different lung cancer tissues in situ, including in AC tissue (30). TGF- $\beta$ is a multifunctional protein and has been demonstrated to be involved in promoting EMT (35). Twist is a basic helix-loop-helix transcription factor and may promote EMT by downregulating E-cadherin, either directly or by interacting with other transcription factors (36). It has also been demonstrated that the SHH pathway is important in the development and metastasis of lung cancer. In a previous study, the expression of Gli1 was associated with the expression of EMT markers E-cadherin and $\beta$-catenin in lung SCC specimens. Inhibition of the SHH/Gli pathway suppressed the migration and upregulation of E-cadherin in lung SCC cells, whereas subsequent stimulation of the SHH pathway increased migration and downregulated the expression of E-cadherin in the lung SCC cells (37). In pancreatic cancer, Xu et al found that five members of the S100 gene family, namely S100A2, S100A4, S100A6, S100A11 and S100A14, were significantly downregulated upon Gli1 knockdown. The migration of pancreatic cancer cells was also significantly increased in a Gli1 expression-dependent manner (38). These results suggest that FAP $\alpha$ may be indirectly involved in the EMT process by regulating certain signaling pathways, including SHH and PI3K. Further investigations are required to investigate the mechanisms involved.

In conclusion, the present study provided evidence that FAP $\alpha$ promoted cellular growth and migration in lung SCC cells, and that the PI3K-AKT and SHH-Glil signaling pathways may be involved in the effects of FAP $\alpha$ on lung SCC cells.

\section{Acknowledgements}

This study was funded by the Beijing Natural Science Foundation of Beijing Municipality, China (grant no. 7132048; Dr Jun Jia) and the Cancer Research Wales and the Albert Hung Foundation (Professor Wen G. Jiang). Dr Jun Jia is a recipient of Cardiff's China Medical Scholarship. 


\section{References}

1. Natarajan J, Chandrashekar C and Radhakrishnan R: Critical biomarkers of epithelial-mesenchymal transition in the head and neck cancers. J Cancer Res Ther 10: 512-518, 2014.

2. Zeisberg M and Neilson EG: Biomarkers for epithelial-mesenchymal transitions. J Clin Invest 119: 1429-1437, 2009.

3. Li ZH and Bresnick AR: The S100A4 metastasis factor regulates cellular motility via a direct interaction with myosin-IIA. Cancer Res 66: 5173-5180, 2006.

4. Sarrió D, Rodriguez-Pinilla SM, Hardisson D, Cano A, Moreno-Bueno G and Palacios J: Epithelial-mesenchymal transition in breast cancer relates to the basal-like phenotype. Cancer Res 68: 989-997, 2008.

5. Scanlan MJ, Raj BK, Calvo B, Garin-Chesa P, Sanz-Moncasi MP, Healey JH, Old LJ and Rettig WJ: Molecular cloning of fibroblast activation protein alpha, a member of the serine protease family selectively expressed in stromal fibroblasts of epithelial cancers. Proc Natl Acad Sci USA 91: 5657-5661, 1994.

6. Huber MA, Kraut N, Park JE, Schubert RD, Rettig WJ, Peter RU and Garin-Chesa P: Fibroblast activation protein: Differential expression and serine protease activity in reactive stromal fibroblasts of melanocytic skin tumors. J Invest Dermatol 120: 182-188, 2003.

7. Kraman M, Bambrough PJ, Arnold JN, Roberts EW, Magiera L, Jones JO, Gopinathan A, Tuveson DA and Fearon DT: Suppression of antitumor immunity by stromal cells expressing fibroblast activation protein-alpha. Science 330: 827-830, 2010.

8. Aertgeerts K, Levin I, Shi L, Snell GP, Jennings A, Prasad GS Zhang Y, Kraus ML, Salakian S, Sridhar V, et al: Structural and kinetic analysis of the substrate specificity of human fibroblast activation protein alpha. J Biol Chem 280: 19441-19444, 2005.

9. Levy MT, McCaughan GW, Abbott CA, Park JE, Cunningham AM, Müller E, Rettig WJ and Gorrell MD: Fibroblast activation protein: A cell surface dipeptidyl peptidase and gelatinase expressed by stellate cells at the tissue remodelling interface in human cirrhosis. Hepatology 29: 1768-1778, 1999.

10. Christiansen VJ, Jackson KW, Lee KN and McKee PA: Effect of fibroblast activation protein and alpha2-antiplasmin cleaving enzyme on collagen types I, III and IV. Arch Biochem Biophys 457: 177-186, 2007.

11. Aggarwal S, Brennen WN, Kole TP, Schneider E, Topaloglu O, Yates M, Cotter RJ and Denmeade SR: Fibroblast activation protein peptide substrates identified from human collagen I derived gelatin cleavage sites. Biochemistry 47: 1076-1086, 2008.

12. O'Brien P and O'Connor BF: Seprase: An overview of an important matrix serine protease. Biochim Biophys Acta 1784: $1130-1145,2008$.

13. Lai D, Ma L and Wang F: Fibroblast activation protein regulates tumor-associated fibroblasts and epithelial ovarian cancer cells. Int J Oncol 41: 541-550, 2012.

14. Wikberg ML, Edin S, Lundberg IV, Van Guelpen B, Dahlin AM, Rutegård J, Stenling R, Oberg A and Palmqvist R: High intratumoral expression of fibroblast activation protein (FAP) in colon cancer is associated with poorer patient prognosis. Tumour Biol 34: 1013-1020, 2013.

15. Saigusa S, Toiyama Y, Tanaka K, Yokoe T, Okugawa Y, Fujikawa H, Matsusita K, Kawamura M, Inoue Y, Miki C, et al: Cancer-associated fibroblasts correlate with poor prognosis in rectal cancer after chemoradiotherapy. Int J Oncol 38: 655-663, 2011.

16. Cohen SJ, Alpaugh RK, Palazzo I, Meropol NJ, Rogatko A, Xu Z, Hoffman JP, Weiner LM and Cheng JD: Fibroblast activation protein and its relationship to clinical outcome in pancreatic adenocarcinoma. Pancreas 37: 154-158, 2008.

17. Jia J, Martin TA, Ye L and Jiang WG: FAP- $\alpha$ (Fibroblast activation protein- $\alpha$ ) is involved in the control of human breast cancer cell line growth and motility via the FAK pathway. BMC Cell Biol 15: 16, 2014

18. Okada K, Chen WT, Iwasa S, Jin X, Yamane T, Ooi A and Mitsumata M: Seprase, a membrane-type serine protease, has different expression patterns in intestinal- and diffuse-type gastric cancer. Oncology 65: 363-370, 2003.

19. Kashyap MK, Marimuthu A, Kishore CJ, Peri S, Keerthikumar S, Prasad TS, Mahmood R, Rao S, Ranganathan P, Sanjeeviah RC, et al: Genomewide mRNA profiling of esophageal squamous cell carcinoma for identification of cancer biomarkers. Cancer Biol Ther 8: 36-46, 2009.
20. Zhang MZ, Qiao YH, Nesland JM, Trope C, Kennedy A, Chen WT and Suo ZH: Expression of seprase in effusions from patients with epithelial ovarian carcinoma. Chin Med J (Engl) 120: 663-668, 2007

21. Iwasa S, Okada K, Chen WT, Jin X, Yamane T, Ooi A and Mitsumata M: 'Increased expression of seprase, a membranetype serine protease, is associated with lymph node metastasis in human colorectal cancer'. Cancer Lett 227: 229-236, 2005.

22. Wang XM, Yu DM, McCaughan GW and Gorrell MD: Fibroblast activation protein increases apoptosis, cell adhesion, and migration by the LX-2 human stellate cell line. Hepatology 42 : 935-945, 2005.

23. Huang Y, Simms AE, Mazur A, Wang S, León NR, Jones B, Aziz N and Kelly T: Fibroblast activation protein- $\alpha$ promotes tumor growth and invasion of breast cancer cells through nonenzymatic functions. Clin Exp Metastasis 28: 567-579, 2011.

24. Wang H, Wu Q, Liu Z, Luo X, Fan Y, Liu Y, Zhang Y, Hua S, Fu Q, Zhao M, et al: Downregulation of FAP suppresses cell proliferation and metastasis through PTEN/PI3K/AKT and Ras-ERK signaling in oral squamous cell carcinoma. Cell Death Dis 5: e1155, 2014

25. Lv B, Xie F, Zhao P, Ma X, Jiang WG, Yu J, Zhang X and Jia J: Promotion of cellular growth and motility is independent of enzymatic activity of fibroblast activation protein- $\alpha$. Cancer Genomics Proteomics 13: 201-208, 2016.

26. Goodman JD, Rozypal TL and Kelly T: Seprase, a membranebound protease, alleviates the serum growth requirement of human breast cancer cells. Clin Exp Metastasis 20: 459-470, 2003.

27. Cheng JD, Dunbrack RL Jr, Valianou M, Rogatko A, Alpaugh RK and Weiner LM: Promotion of tumor growth by murine fibroblast activation protein, a serine protease, in an animal model. Cancer Res 62: 4767-4772, 2002.

28. Huang Y, Wang S and Kelly T: Seprase promotes rapid tumor growth and increased microvessel density in a mouse model of human breast cancer. Cancer Res 64: 2712-2716, 2004.

29. Yang L, Ma L and Lai D: Over-expression of fibroblast activation protein alpha increases tumor growth in xenografts of ovarian cancer cells. Acta Biochim Biophys Sin (Shanghai) 45: 928-937, 2013.

30. Du H, Chen D, Zhou Y, Han Z and Che G: Fibroblast phenotypes in different lung diseases. J Cardiothorac Surg 9: 147, 2014

31. Yang W, Han W, Ye S, Liu D, Wu J, Liu H, Li C and Chen H: Fibroblast activation protein- $\alpha$ promotes ovarian cancer cell proliferation and invasion via extracellular and intracellular signaling mechanisms. Exp Mol Pathol 95: 105-110, 2013.

32. Chung KM, Hsu SC, Chu YR, Lin MY, Jiaang WT, Chen RH and Chen X: Fibroblast activation protein (FAP) is essential for the migration of bone marrow mesenchymal stem cells through RhoA activation. PLoS One 9: e88772, 2014.

33. Zi FM, He JS, Li Y, Wu C, Wu WJ, Yang Y, Wang LJ, He DH, Yang L, Zhao Y, et al: Fibroblast activation protein protects bortezomib-induced apoptosis in multiple myeloma cells through $\beta$-catenin signaling pathway. Cancer Biol Ther 15: 1413-1422, 2014.

34. Henriksson ML, Edin S, Dahlin AM, Oldenborg PA, Öberg Å, Van Guelpen B, Rutegård J, Stenling R and Palmqvist R: Colorectal cancer cells activate adjacent fibroblasts resulting in FGF1/FGFR3 signaling and increased invasion. Am J Pathol 178: 1387-1394, 2011.

35. Katsuno Y, Lamouille $S$ and Derynck R: TGF- $\beta$ signaling and epithelial-mesenchymal transition in cancer progression. Curr Opin Oncol 25: 76-84, 2013.

36. Sánchez-Tilló E, Liu Y, de Barrios O, Siles L, Fanlo L, Cuatrecasas M, Darling DS, Dean DC, Castells A and Postigo A: EMT-activating transcription factors in cancer: Beyond EMT and tumor invasiveness. Cell Mol Life Sci 69: 3429-3456, 2012.

37. Yue D, Li H, Che J, Zhang Y, Tseng HH, Jin JQ, Luh TM, Giroux-Leprieur E, Mo M, Zheng Q, et al: Hedgehog/Gli promotes epithelial-mesenchymal transition in lung squamous cell carcinomas. J Exp Clin Cancer Res 33: 34, 2014.

38. Xu X, Su B, Xie C, Wei S, Zhou Y, Liu H, Dai W, Cheng P, Wang F, Xu X, et al: Sonic hedgehog-Gli1 signaling pathway regulates the epithelial mesenchymal transition (EMT) by mediating a new target gene, S100A4, in pancreatic cancer cells. PLoS One 9: e96441, 2014.

This work is licensed under a Creative Commons Attribution-NonCommercial-NoDerivatives 4.0 International (CC BY-NC-ND 4.0) License. 